\title{
Terapia nutricional enteral em politraumatizados sob ventilação mecânica e oferta energética
}

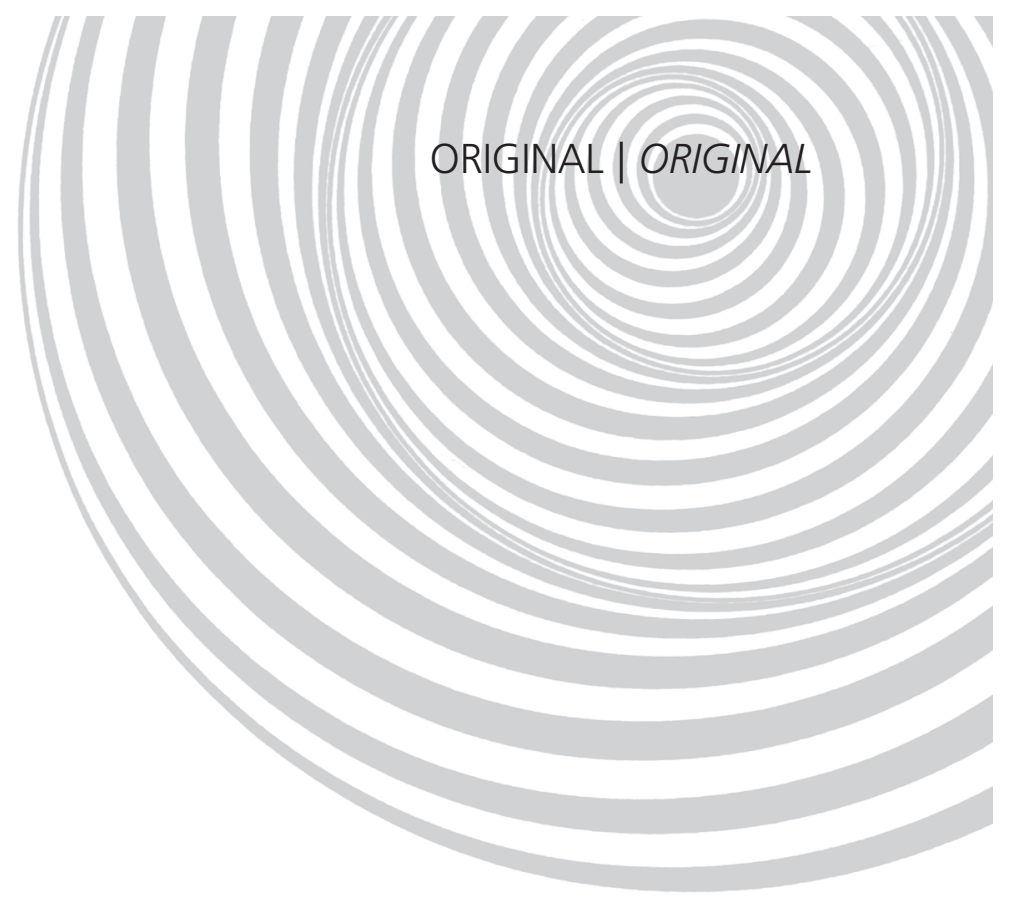

\author{
Enteral nutritional therapy in \\ mechanically-ventilated multiple-trauma \\ patients and energy intake
}

Cecília Flávia Lopes COUTO'

José da Silva MOREIRA ${ }^{1}$

Jorge Amilton HOHER²

RE S U M O

\section{Objetivo}

O objetivo deste estudo foi avaliar a adequação energética dos pacientes politraumatizados em suporte ventilatório internados na unidade de terapia intensiva de um hospital público de Porto Alegre (RS), por meio da comparação entre as calorias prescritas e as efetivamente administradas, assim como entre as calorias estimadas pela equação de Harris-Benedict e a prescrição energética de cada paciente.

\section{Métodos}

Estudo de coorte prospectivo de pacientes politraumatizados, simultaneamente sob ventilação mecânica e terapia nutricional enteral. Verificou-se o tempo de permanência sob ventilação mecânica e a oferta energética durante o período de terapia nutricional enteral. A associação entre as variáveis quantitativas foi avaliada através do teste de correlação de Spearman devido à assimetria das variáveis.

\section{Resultados}

Foram acompanhados 60 pacientes, na faixa etária de 18 a 78 anos, sendo 81,7\% do sexo masculino. Os tempos medianos de internação hospitalar, permanência na unidade de terapia intensiva e ventilação mecânica foram de 29, 14 e 6 dias, respectivamente. A média do percentual de dieta administrada foi de $68,6 \%$ ( $\mathrm{DP}=18,3 \%$ ). Da amostra total, 16 (26,7\%) pacientes receberam no mínimo 80\% de suas necessidades diárias. Não houve associação estatisticamente significativa entre o valor energético total administrado e os tempos de ventilação mecânica $\left(r_{s}=0,130 ; p=0,321\right)$, de unidade de terapia intensiva $\left(r_{s}=-0,117 ; p=0,372\right)$ e de internação hospitalar $\left(r_{s}=-0,152 ; p=0,246\right)$.

\footnotetext{
1 Universidade Federal do Rio Grande do Sul, Faculdade de Medicina, Programa de Pós-Graduação em Ciências Pneumológicas. R. Ramiro Barcelos, 2400, $2^{\circ}$ andar, Bom Fim, 90035-003, Porto Alegre, RS, Brasil. Correspondência para/Correspondence to: CFL COUTO.E-mail: <cecilialcouto@hotmail.com>.

${ }^{2}$ Universidade Federal de Ciências da Saúde de Porto Alegre, Curso de Nutrição. Porto Alegre, RS, Brasil.
} 
696 | CFL COUTO et al.

\section{Conclusão}

Os pacientes incluídos neste estudo não receberam com precisão o aporte energético prescrito, ficando expostos aos riscos da desnutrição e seus desfechos clínicos desfavoráveis.

Termos de indexação: Ingestão de energia. Necessidade energética. Nutrição enteral. Respiração artificial. Traumatismo múltiplo.

\section{A B S T R A C T}

\section{Objective}

The objective of this study was to asssess the adequacy of energy intake of multiple trauma patients in the intensive care unit of a public hospital in the city of Porto Alegre, Southern Brazil, who require ventilatory support, by comparing prescribed energy intake with effectively administered energy, and energy requirement estimated by the Harris-Benedict equation with the energy prescription of each patient.

\section{Methods}

This is a prospective cohort study of patients with multiple trauma simultaneously on mechanical ventilation and enteral nutrition. Duration of mechanical ventilation and energy intake during enteral nutrition were verified. The association between quantitative variables was assessed by the Spearman correlation test due to variable asymmetry.

\section{Results}

Sixty patients aged 18 to 78 years were studied, $81.7 \%$ of them males. Median length of hospital stay, intensive care unit stay, and duration of mechanical ventilation was 29, 14, and 6 days, respectively. The mean percentage of administered calories was $68.6 \%$ (SD=18.3\%). Of the entire sample, 16 (26.7\%) patients received at least $80 \%$ of their daily energy requirement. There was no significant association between total energy administered and duration of mechanical ventilation $\left(r_{s}=0.130 ; p=0.321\right)$, length of intensive care unit stay $\left(r_{s}=-0.117\right.$; $p=0.372)$ and length of hospital stay $\left(r_{s}=-0.152 ; p=0.246\right)$.

\section{Conclusion}

The study patients did not receive the prescribed energy. Therefore, they were at risk of malnutrition and its adverse clinical outcomes.

Indexing terms: Energy intake. Energy requirement. Enteral nutrition. Artificial respiration. Multiple trauma.

\section{N T R O D U ÇÃ O}

O estado de desnutrição proteico-energética ocorre quando as necessidades do organismo por proteínas e calorias como combustível energético não conseguem ser devidamente supridas pela dieta ${ }^{1}$. Pacientes graves apresentam resposta metabólica intensa, que se caracteriza por aumento no catabolismo energético. Essa situação é ainda mais preocupante durante a fase aguda da doença, quando os pacientes são expostos à subnutrição resultante da administração inadequada de nutrientes, que pode levar à desnutrição e a suas consequências².

A ocorrência de desnutrição ocasiona depressão da resposta imunológica, comprometimento da cicatrização e diminuição das forças musculares, aumentando a probabilidade de ocorrência de infecções e de outras complicações clínicas em pacientes hospitalizados. Além disso, pode resultar em prolongamento do tempo de permanência no hospital, aumento da mortalidade e também do custo hospitalar ${ }^{3}$. Entre 43\% e 88\% dos pacientes críticos internados em Unidade de Terapia Intensiva (UTI) apresentam desnutrição proteico-energética, configurando-se, assim, como um problema frequente e prevalente, principalmente nos pacientes ventilados mecanicamente ${ }^{4-6}$.

Pacientes com traumatismo múltiplo, em terapia intensiva, encontram-se em estado hipermetabólico, no qual se faz necessário um aporte calórico adequado e suficiente para atender ao 
intenso gasto energético do organismo nesse período. Em caso de aporte inadequado, as reservas de aminoácidos dos músculos esqueléticos e respiratórios são recrutadas, expondo o paciente crítico a um maior risco nutricional e complicações pulmonares ${ }^{7,8}$. A desnutrição nos pacientes politraumatizados determina depressão da resposta imunológica, retarda a cicatrização de feridas, dificulta a regeneração do epitélio respiratório, enfraquece o músculo respiratório e, consequentemente, determina o prolongamento do tempo de Ventilação Mecânica (VM).

Sabe-se que pacientes que permanecem por mais tempo sob VM apresentam um pior desfecho clínico, maior tempo de permanência hospitalar e alto índice de infecções e de mortalidade ${ }^{7,9}$. No entanto, um aporte calórico adequado pode promover o desmame da VM em pacientes críticos, reduzindo o tempo de internação hospitalar e a ocorrência de infecções ${ }^{10}$.

Estudos relatam que 50 a $90 \%$ das necessidades energéticas prescritas são efetivamente administradas aos pacientes. Essa discrepância pode ser decorrente de fatores previsíveis na UTI, como suspensões periódicas das dietas para procedimentos diagnósticos e terapêuticos e para cuidados com as vias aéreas, e disfunções do trato digestório, que dificultam a adequada administração da nutrição enteral ${ }^{2,11,12}$.

Uma grave consequência da inadequada administração de nutrientes ao paciente crítico em VM é a desnutrição. O estudo de McClave et al. ${ }^{12}$, realizado em 1998, concluiu que deve ser considerada subalimentação a administração calórica menor que $90 \%$ do estimado, o que acarretaria deficit nutricional e suas complicações ${ }^{13}$.

Diante desse cenário, é importante estimar com a maior precisão possível as necessidades energéticas desses pacientes e analisar com frequência se a quantidade correta de nutrientes, inicialmente prevista, foi realmente administrada.

Este estudo teve por objetivo avaliar a adequação energética dos pacientes em suporte ventilatório internados na UTI de um hospital púbico de Porto Alegre (RS), por meio da comparação entre as calorias prescritas e as efetivamente administradas, assim como entre as calorias estimadas pela equação de Harris-Benedict e a prescrição energética de cada paciente.

\section{MÉ TO D OS}

Estudo de coorte, prospectivo, observacional, em que foram incluídos 60 pacientes politraumatizados, com idade igual ou superior a 18 anos, que se encontravam simultaneamente sob VM e terapia nutricional enteral, com período de internação em UTI superior a cinco dias. A coleta de dados foi realizada na UTI do Hospital Cristo Redentor, Grupo Hospitalar Conceição de Porto Alegre, no período de abril de 2008 a junho de 2009.

O estudo foi aprovado pelo Comitê de Ética em Pesquisa do Hospital Nossa Senhora da Conceição - Grupo Hospitalar Conceição, Unidade Hospital Cristo Redentor, Protocolo n ${ }^{\circ}$ 023/08, em 12 de março de 2008, e todos os responsáveis legais dos participantes assinaram um termo de consentimento livre e esclarecido antes de sua inclusão na amostra, devido ao impedimento dos pacientes em VM de assinar por estarem sedados e com baixo nível de consciência.

Os critérios para inclusão no estudo foram: pacientes com diagnóstico de traumatismo múltiplo decorrente de acidente de trânsito ou queda de altura, que se encontravam simultaneamente sob VM e terapia nutricional enteral, com um período de internação em UTI de no mínimo cinco dias. Nenhum paciente foi submetido a cirurgia durante o período de observação do estudo, o que seria considerado critério de exclusão.

Foram verificados o tempo em que os pacientes $(n=60)$ permaneceram sob VM e a quantidade de calorias por eles recebidas durante o período em que se encontravam em suporte nutricional enteral.

As necessidades nutricionais de cada paciente foram determinadas por meio de medidas 
antropométricas, como estatura recumbente, altura do joelho e peso ideal, utilizado por não se dispor de cama balança para obtenção do peso atual. Para o cálculo do peso ideal, primeiramente foi estimada a altura do indivíduo através da equação por meio da altura do joelho preconizada por Chumlea et al. ${ }^{14}$. Após ser estimada a altura do paciente, estimou-se a compleição corporal e verificou-se o peso ideal do paciente na tabela de referência de peso adaptada do Metropolitan Life Insurance. O Gasto Energético Total (GET) foi determinado pela equação de Harris-Benedict ${ }^{15}$, multiplicando-se o Gasto Energético Basal (GEB) pelo fator de atividade e fator de injúria ${ }^{16}$. Foi utilizado 1,5 como fator de injúria, o qual corresponde a trauma e infecção moderada, e 1,10 para o fator de atividade, valor correspondente a pacientes acamados sob VM.

A partir da estimativa do Gasto Energético Basal, foi determinada a fórmula enteral, de acordo com o protocolo da instituição (dieta polimérica padrão, com densidade energética de $1,2 \mathrm{kcal} / \mathrm{mL}$, e dieta para diabetes ou hipossódica e para diarreia, com densidade energética de 1,06kcal $/ \mathrm{mL}$ ), que foi administrada por sonda nasoentérica, com velocidade de infusão da dieta ajustada progressivamente de acordo com a aceitação do paciente, evoluindo em dois dias até atingir a meta nutricional. Com base na revisão bibliográfica, utilizou-se o estudo de Assis et al. ${ }^{17}$ como referência para o ponto de corte de $80 \%$ como objetivo nutricional, considerando-se, assim, como adequada uma administração ernegética superior a $80 \%$ das necessidades totais diárias. Portanto, foi realizada uma comparação entre o grupo de pacientes que recebeu menos que $80 \%$ do volume prescrito e o grupo de pacientes que recebeu $80 \%$ ou mais do volume prescrito.

No acompanhamento diário, foram realizadas aferições do volume (em mililitros) de dieta enteral administrado em cada indivíduo, que foi posteriormente convertido em calorias para ser comparado com as necessidades energéticas totais calculadas e com cada prescrição diária. Dessa forma, foi calculado o percentual de calorias administradas [(calorias administradas/calorias prescritas)*100] para cada dia, totalizando um mínimo de cinco aferições desse percentual. Com o propósito de resumir os valores encontrados em um único valor para inclusão no banco de dados, foi estudada a distribuição da variável e observou-se que o percentual de calorias administradas ao paciente durante o período de internação na UTI apresentou distribuição assimétrica. A assimetria foi consequência dos dias em que alguns pacientes se encontravam em jejum. Devido a essa condição, a medida de tendência central escolhida para resumir os dados foi a mediana.

Após o cálculo das medianas para cada paciente, foram realizadas estatísticas descritivas para essa variável e observou-se que ela apresentava distribuição simétrica. Dessa forma, a medida de tendência central utilizada para descrever o percentual de calorias administradas foi a média, e a medida de dispersão utilizada foi o desvio-padrão.

As demais variáveis quantitativas (idade, prescrição energética, administração calórica, GET, Acute Physiology and Chronic Health II (APACHE II), tempo de VM, tempo de internação na UTI, tempo de internação hospitalar) foram descritas através de médias e desvios-padrão (distribuição simétrica) ou mediana e amplitude interquartílica (distribuição assimétrica).

As variáveis qualitativas (sexo, causa do politraumatismo, classificação do percentual de dieta administrada em $<80 \%$ ou $\geq 80 \%$ ) foram expressas através de frequências absolutas e relativas e comparadas por meio do teste Qui-quadrado de Pearson. Para avaliar a associação entre as variáveis quantitativas, utilizou-se o teste de correlação de Spearman devido à assimetria das variáveis envolvidas na análise.

O teste $t$ de Student foi empregado na comparação do percentual do valor energético total administrado entre os pacientes que receberam dieta enteral ou ficaram em jejum em algum momento. Foi calculada a mediana do percentual do valor energético total administrado devido à discrepância entre os dias (coeficiente de variação $=43 \%$ ). 
O nível de significância adotado foi de 5\%, e as análises estatísticas foram realizadas no programa Statistical Package for the Social Sciences (SPSS), versão 13.0.

\section{RES U LTA D OS}

Foram acompanhados 60 pacientes, sendo $49(81,7 \%)$ do sexo masculino e $11(18,3 \%)$ do sexo feminino, com média de idade de 39, 5 anos. O estudo evidenciou como causa de trauma múltiplo acidente de trânsito $(91,7 \%)$, seguido de queda de altura (8,3\%). A média do APACHE ॥ foi 12,1 .

O tempo de internação hospitalar foi, em mediana, de 29 dias, e o tempo mediano de internação na UTI, de 14 dias. O tempo de permanência sob VM, em mediana, foi de 6 dias (Tabela 1). O número de dias em que os pacientes receberam dieta enteral, em mediana, foi de 11,5 dias.

A média do percentual de dieta administrada foi de 68,6\% (Desvio-Padrão-DP=18,3\%). Da amostra total, 9 (15,0\%) pacientes receberam menos da metade do que deveria ser administrado, e apenas $16(26,7 \%)$ receberam quantidade correspondente ao mínimo de $80,0 \%$ de suas necessidades diárias (Figura 1).

Quando avaliado o percentual de dieta administrada, houve diferença estatisticamente significativa $(p=0,045)$ entre os pacientes. Os 18 pacientes que tiveram, em algum momento, sua dieta suspensa, apresentaram média de administração calórica significativamente menor $(61,4 \%)$ em relação aos 42 que receberam dieta todos os dias $(71,7 \%)$. Destes, $38(90,5 \%)$ tiveram uma administração de, no mínimo, 50,0\% da dieta prescrita. Treze pacientes ( $72,2 \%)$ tiveram a dieta suspensa por pelo menos um dia.

Comparando-se os pacientes que receberam menos de $80 \%$ do valor energético prescrito com os que receberam acima desse valor, não houve diferença estatisticamente significativa $(p=0,137)$ entre os grupos. Entretanto, observou-se que os pacientes com percentual de administração inferior a $80 \%$ da dieta prescrita apresentavam maior tempo de internação e idade levemente superior $(p=0,068)$ (Tabela 1$)$. Ao avaliar a associação entre idade e o percentual de dieta administrada, através do coeficiente de correlação de Pearson, verificou-se correlação inversa estatisticamente significativa entre essas variáveis $(r=-0,300 ; p=0,020)$, ou seja, pacientes mais velhos tendem a receber um menor percentual de administração de dieta (Figura 2).

Não houve associação estatisticamente significativa entre o percentual de calorias administradas e os tempos de VM $\left(r_{s}=0,130 ; p=0,321\right)$, de permanência em UTI $\left(r_{s}=-0,117 ; p=0,372\right)$ e de internação hospitalar $\left(r_{s}=-0,152 ; p=0,246\right)$.

Tabela 1. Características demográficas e clínicas dos pacientes na amostra total e de acordo com o percentual de calorias administradas. Hospital Cristo Redentor, Porto Alegre (RS), 2008-2009.

\begin{tabular}{|c|c|c|c|c|}
\hline Variáveis ${ }^{*}$ & $\begin{array}{l}\text { Amostra total } \\
\qquad(n=60)\end{array}$ & $\begin{array}{c}<80 \% \text { do prescrito } \\
(n=44)\end{array}$ & $\begin{array}{l}\geq 80 \% \text { do prescrito } \\
\quad(n=16)\end{array}$ & valor-p \\
\hline Idade (anos) & $M=39,5, D P=17,3$ & $M=41,5, D P=17,8$ & $M=34,0, D P=14,9$ & 0,137 \\
\hline Sexo masculino & $49(81,7)$ & $34(77,3)$ & $15(93,8)$ & 0,259 \\
\hline Traumatismo por acidente de trânsito & $55(91,7)$ & $40(90,9)$ & $15(93,8)$ & 1,000 \\
\hline APACHE II & $M=12,1, D P=5,2$ & $M=12,4, D P=5,4$ & $M=11,0, D P=4,5$ & 0,349 \\
\hline Tempo VM (dias) & $6(5-11)$ & $6(4,3-11)$ & $6,5(5-13)$ & 0,699 \\
\hline Tempo UTI (dias) & $14(11-19)$ & $14(11-20)$ & $13,5(12-18)$ & 0,814 \\
\hline Tempo internação (dias) & $29(21-39)$ & $33,5(24,3-42,8)$ & $25(19,3-32)$ & 0,068 \\
\hline Tempo de uso de dieta enteral (dias) & $11,5(9-15)$ & $11,5(8,3-15)$ & $11,5(10-14,8)$ & 0,867 \\
\hline
\end{tabular}

APACHE II: Acute Physiology and Chronic Health Evaluation; UTI: Unidade de Terapia Intensiva; VM: Ventilação Mecânica; M: Média; DP: Desvio-Padrão.

* Variáveis quantitativas estão expressas em média, desvio-padrão ou mediana (percentis 25 - 75) quando apropriado; variáveis categóricas estão descritas por $\mathrm{n}(\%)$. 
700 | CFL COUTO et al.

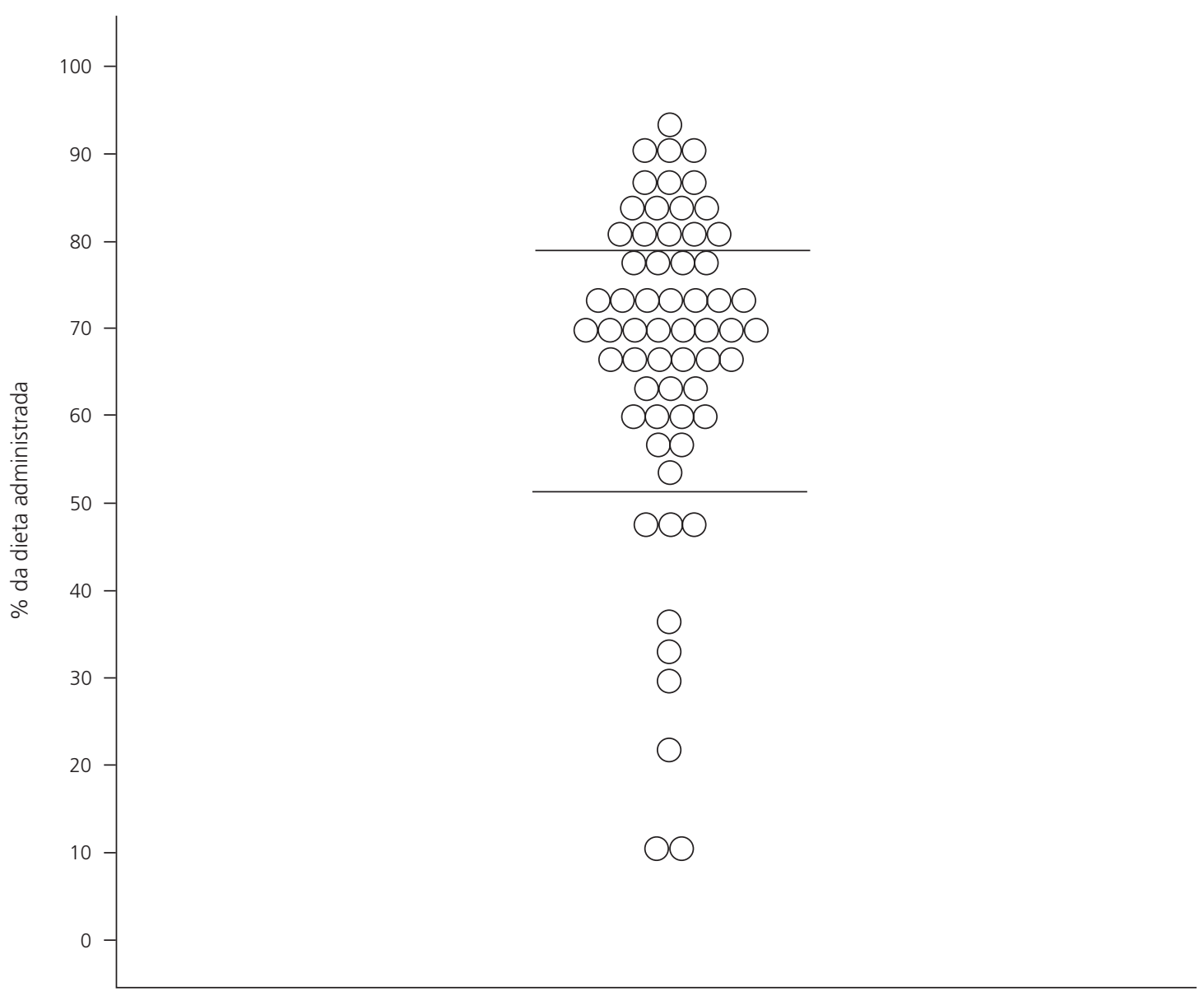

Figura 1. Distribuição da amostra em relação ao percentual de dieta administrada conforme pontos de corte (50\% e $80 \%$ ).

Do total de pacientes ( $n=60), 91,2 \%$ tiveram prescrição coerente com o GET estimado pela equação de Harris-Benedict. O valor médio do GET calculado por paciente foi de $2.515,6 \mathrm{kcal}$, e a quantidade média das calorias ofertadas foi de $1.365 \mathrm{kcal} /$ por dia por paciente, que corresponde em média a $20 \mathrm{kcal} / \mathrm{kg}$ peso.

Não houve associação entre o percentual de dieta administrada e o número de dias em que os pacientes receberam dieta por sonda enteral $\left(r_{s}=-0,057 ; p=0,663\right)$.

\section{I S C U S S Ã O}

Este estudo revelou uma discrepância entre o valor calórico prescrito e o administrado, haja vista que os pacientes receberam em média
$68,6 \%$ do que foi prescrito. Observou-se, também, divergências entre os valores energéticos prescritos e o GET estimado a partir da equação de Harris-Benedict, método amplamente utilizado como parâmetro de estimativa das necessidades energéticas de pacientes hospitalizados ${ }^{18,19}$.

Teixeira et $a l .^{3}$, em estudo com pacientes de UTI, avaliaram a adequação da terapia nutricional dessa unidade e relataram que $74,4 \%$ das calorias foram efetivamente administradas em relação ao prescrito. Kyle et al. ${ }^{20}$ relataram que $26,0 \%$ dos pacientes prospectados para a pesquisa tiveram uma administração calórica igual ou superior a $66,6 \%$ quando comparada às necessidades energéticas, considerando 20 a $25 \mathrm{kcal} / \mathrm{kg}$ de peso para mulheres e 25 a $30 \mathrm{kcal} / \mathrm{kg}$ de peso para homens. Whelan et al. ${ }^{21}$, por sua vez, observaram 

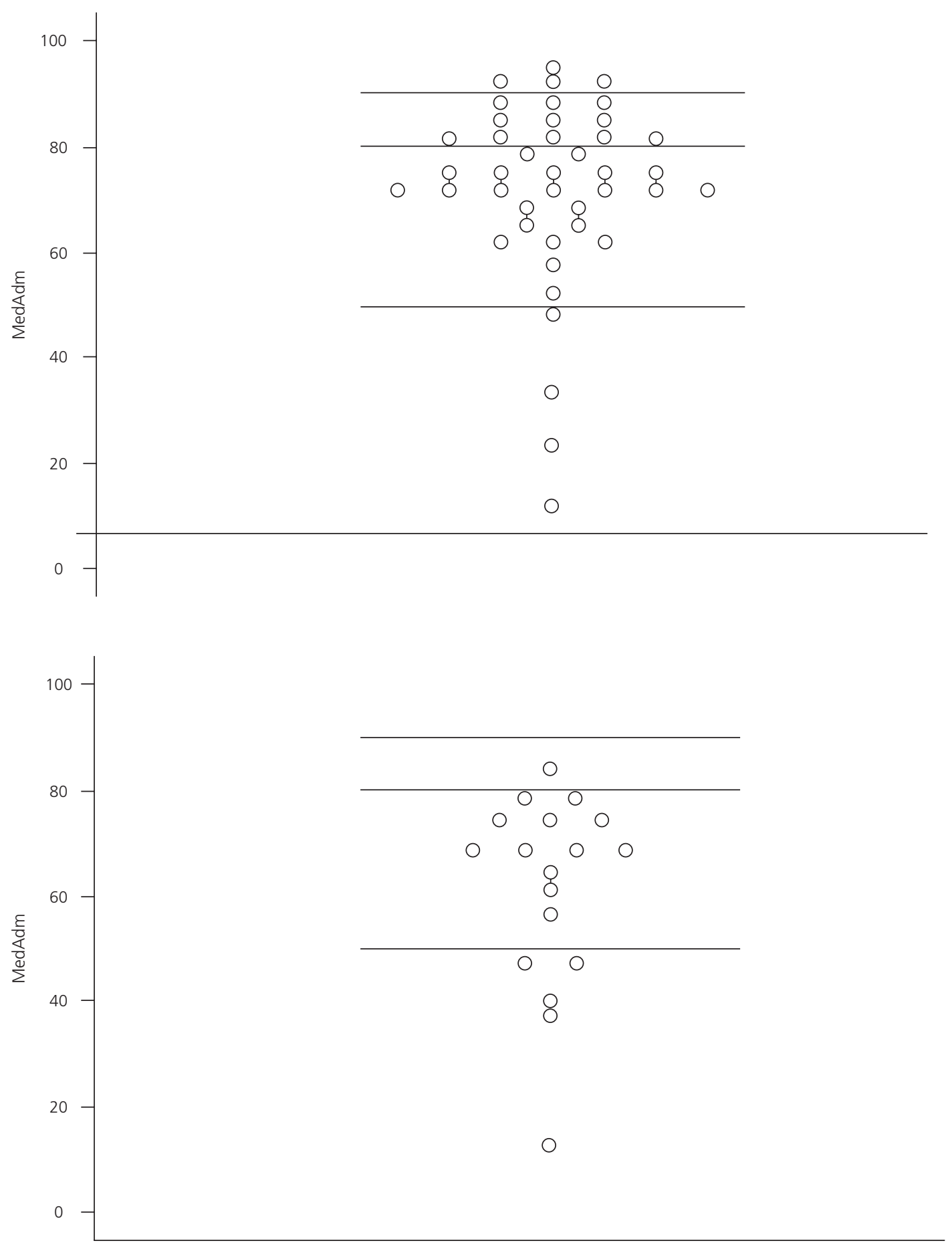

Figura 2. Percentual de dieta enteral administrada de acordo com os pontos de corte de $50 \%, 80 \%$ e $90 \%$ encontrados na literatura como o mínimo a ser administrado.

uma média de administração de 80,0\%. De Jonghe et al. ${ }^{2}$ constataram que ape-nas $71,2 \%$ das necessidades energéticas dos pacientes incluídos no estudo foram efetivamente administradas. O'Leary et al. ${ }^{22}$ relataram que mais da metade dos indivíduos investigados receberam 
uma quantidade energética inferior a 50,0\% das necessidades energéticas.

Conforme estudo de McClave et al. ${ }^{12}$, a inadequação energética é classificada como subalimentação quando a administração for menor que 90,0\% das necessidades energéticas; como adequada, entre 90,0 e $110,0 \%$; e como superalimentação quando acima de $110,0 \%$. Tomando como base esse ponto de corte de $90,0 \%$, $56,7 \%$ desses pacientes apresentariam uma prescrição calórica menor que 90,0\% do GET estimado, e 18,3\% uma prescrição acima de $110,0 \%$. Dessa forma, apenas $25,0 \%$ dos pacientes estariam recebendo prescrição calórica adequada (entre $90,0 \%$ e $110,0 \%$ ).

Uma grave consequência da inadequada administração de nutrientes é o fato de o paciente crítico em VM ficar subalimentado, ou seja, receber uma quantidade energética menor do que as suas necessidades, o que poderá conduzir o paciente ao estado de desnutrição. Segundo Giner et al. ${ }^{23}$, a desnutrição e suas consequências estão presentes nos pacientes internados em UTI com uma estimativa de ocorrência acima de 40\%. Marshall \& West $^{24}$ também evidenciaram que um número significativo de pacientes não tem suas necessidades nutricionais efetivamente administradas, recebendo, em geral, uma quantidade inferior às suas necessidades.

O estudo de Faisy et al. ${ }^{25}$ demonstrou uma associação entre o balanço energético negativo e a mortalidade em UTI de pacientes gravemente doentes que permaneceram por tempo prolongado sob VM enquanto recebiam nutrição enteral exclusiva. No estudo realizado por Kan et al. ${ }^{10}$, os pacientes que receberam uma alimentação inadequada para suprir a demanda energética dependeram por mais tempo da VM. O presente estudo não encontrou associação estatisticamente significativa entre o percentual de calorias administradas e os tempos de VM $\left(r_{s}=0,130 ; p=0,321\right)$, de permanência em UTI $\left(r_{s}=-0,117 ; p=0,372\right)$ e de internação hospitalar $\left(r_{s}=-0,152 ; p=0,246\right)$.

A subalimentação, tal como a superalimentação, apresenta efeito deletério ao organis- mo. Uma administração energética insuficiente para suprir a demanda energética ocasiona desnutrição e suas consequências, além do aumento da permanência hospitalar ${ }^{26}$. Ocorrem, também, diminuição da função muscular respiratória e diminuição da resposta imunológica ${ }^{27}$. Cheng et al. ${ }^{4}$ demonstram que nutrir os pacientes críticos sob VM com uma quantidade energética inferior ao necessário dificulta o desmame, o que resulta em complicação, como, por exemplo, infecção.

Utilizando o estudo de Assis et al. ${ }^{17}$ como referência para o ponto de corte de $80,0 \%$, apresentado como objetivo nutricional, este estudo observou que somente $26,7 \%$ dos pacientes receberam as calorias adequadas, ou seja, a quantidade mínima necessária para diminuir as chances de morte em UTI e a mortalidade hospitalar ${ }^{2,28}$. Se se considerar o ponto de corte de $90,0 \%$ proposto por McClave et al. ${ }^{12}$ como objetivo nutricional, somente $6,7 \%$ dos pacientes receberam a quantidade energética adequada.

Sabe-se que condições relacionadas à rotina da UTI, tais como intolerância gastrointestinal (diarreia, vômito) e jejum ou interrupção da administração da dieta para a realização de procedimentos diagnósticos e terapêuticos, podem influenciar na administração adequada da nutrição entera| ${ }^{29-32}$. Binnekade et al. ${ }^{33}$ relataram a incidência de perda na motilidade intestinal e esvaziamento gástrico lento que ocorre em 50 a $60 \%$ dos pacientes críticos, interferindo na tolerância alimentar e ocasionando uma administração da dieta enteral inferior ao previsto. A maioria dos pacientes em VM necessita de sedação e analgesia, e muitos medicamentos utilizados para esse fim, como os opioides, prejudicam a motilidade do trato gastrointestinal e dificultam ainda mais a administração adequada de nutrientes $^{34}$. No presente estudo, entretanto, não foram investigadas as causas das interrupções da infusão da dieta.

Outra situação a ser considerada é a superalimentação, que acontece pelo cálculo superestimado do GET e que pode levar o paciente 
a desenvolver alterações metabólicas e respiratórias, como hipercapnia, aumento do tempo de VM, esteatose hepática, hiperglicemia, acidose metabólica, hipertrigliceridemia e síndrome de realimentação ${ }^{35,36}$.

O gasto energético pode ser estimado por fórmulas e nomogramas ou medido por meio de calorimetria indireta. O presente estudo utilizou a equação de Harris-Benedict, que, embora muito questionada em relação a sua precisão, é frequentemente utilizada no meio hospitalar devido à indisponibilidade de monitor específico para a calorimetria indireta ${ }^{8}$.

Alguns estudos relatam que a equação de Harris-Benedict, principalmente em pacientes críticos, poderia superestimar a necessidade energética, levando à superalimentação e a complicações metabólicas e respiratórias ${ }^{11,18}$. Por esse motivo, muitos profissionais optam por prescrever uma quantidade inferior de calorias e evoluir cuidadosamente a dieta.

Atualmente, fórmulas que consideram 20-30 kcal/kg de peso/dia (recomendação das diretrizes europeias para terapia intensiva) ${ }^{37}$ vêm sendo cada vez mais utilizadas para estimar o gasto energético dos pacientes críticos devido à facilidade de cálculo. No entanto, essas fórmulas não foram utilizadas no presente estudo, pois a equação de Harris-Benedict faz parte da rotina nutricional da unidade onde se desenvolveu a pesquisa.

A calorimetria indireta é considerada o método mais preciso para a determinação das necessidades energéticas dos pacientes sob VM ${ }^{11}$. Entretanto, esse método também apresenta limitações por depender de um monitor de gases e por apresentar dificuldade de aplicação em casos de pacientes críticos que se encontram em instabilidade hemodinâmica ${ }^{38}$. Portanto, foram limitações deste estudo a utilização da equação de Harris-Benedict para estimar o gasto energético dos pacientes e a não inclusão da análise do aporte proteico.
Desse modo, de acordo com os conceitos de subalimentação (<90\% do GET), alimentação adequada (90-110\% do GET) e superalimentação (>110\% do GET) $)^{12}$, o presente estudo demonstrou que $25 \%$ dos pacientes investigados receberam prescrição adequada. A maioria dos pacientes (75\%), no entanto, não apresentou prescrição adequada por ser consenso que a equação de Harris-Benedict superestima em 10\% a 15\% o $\mathrm{GET}^{39}$.

\section{O N C L U S Ã O}

Os pacientes com traumatismo múltiplo sob ventilação mecânica incluídos neste estudo não receberam com precisão o aporte energético prescrito, ficando, assim, expostos aos riscos da desnutrição e a seus desfechos clínicos desfavoráveis.

O presente estudo demonstrou que somente $26,7 \%$ dos pacientes receberam $80,0 \%$ ou mais de suas necessidades energéticas. A maioria, $73,3 \%$, recebeu aporte energético inferior ao adequado. De acordo com os dados analisados, não há correlação entre a administração energética inadequada e o tempo de ventilação mecânica.

\section{COLABORADORES}

CFL COUTO foi responsável pela execução da coleta de dados, trabalhou diretamente com a revisão bibliográfica e redação do artigo. JS MOREIRA contribuiu com as conclusões do trabalho. JA HOHER orientou e trabalhou diretamente com a revisão bibliográfica e com a redação do texto do artigo.

\section{REFERÊ N CIAS}

1. Villet $S$, Chiolero RL, Bollmann MD, Revelly JP, Cayeux RNM, Delarue J, et al. Negative impact of hypocaloric feeding and energy balance on clinical outcome in ICU patients. Clin Nutr. 2005; 24(4): 502-9. doi: 10.1016/j.clnu.2005.03.006.

2. De Jonghe B, Appere-De-Vechi C, Fournier M, Tran B, Merrer J, Melchior JC, et al. A prospective survey 
of nutritional support practices in intensive care unit patients: what is prescribed? What is delivered? Crit Care Med. 2001; 29(1):8-12. doi: 10.1097/00 003246-200101000-00002.

3. Teixeira AC, Caruso L, Soriano FG. Terapia nutricional enteral em unidade de terapia intensiva: infusão versus necessidade. Rev Bras Ter Intens. 2006; 18:331-7.

4. Cheng $\mathrm{CH}$, Chen $\mathrm{CH}$, Wong $\mathrm{Y}$, Lee BJ, Kan MN, Huang YC. Measured versus estimated energy expenditure in mechanically ventilated critically ill patients. Clin Nutr. 2002; 21(2):165-72. doi: 10.10 54/clnu.2002.0526.

5. Krishnan JA, Parce PB, Martinez A, Diette GB, Brower RG. Caloric intake in medical ICU patients: consistency of care with guidelines and relationship to clinical outcomes. Chest. 2003; 124(1):297-305. doi: 10.1378/chest.124.1.297.

6. Waitzberg DL, Caiaffa WT, Correia MI. Hospital malnutrition: the Brazilian national survey (IBRANUTRI): a study of 4000 patients. Nutrition. 2001; 17(7-8):573-80. doi: 10.1016/50899-90 07(01)00573-1.

7. Rasslan S, Candelárias P. Trauma. In: Waittzberg $\mathrm{DL}$, editor. Nutrição oral, enteral e parenteral na prática clínica. $3^{a}$ ed. São Paulo: Atheneu; 2006.

8. Wray CJ, Mammen JM, Hasselgren PO. Catabolic response to stress and potential benefits of nutrition support. Nutrition. 2002; 18(11-12):971-7. doi: S0 899900702009851.

9. Nozaki VT, Peralta RM. Adequação do suporte nutricional na terapia nutricional enteral: uma comparação em 2 hospitais. Rev Nutr. 2009; 22(3): 341-50. doi: 10.1590/S1415-52732009000300 004.

10. Kan MN, Chang HH, Sheu WF, Cheng CH, Lee BJ, Huang YC. Estimation of energy requirements for mechanically ventilated, critically ill patients using nutritional status. Crit Care. 2003; 7(5):R108-15. doi: 10.1186/cc2366

11. Adam S, Batson S. A study of problems associated with the delivery of enteral feed in critically ill patients in five ICUs in the UK. Intensive Care Med. 1997; 23(3):261-6. doi: 10.1007/s001340050326.

12. McClave SA, Lowen CC, Kleber MJ, Nicholson JF, Jimmerson SC, McConnell JW, et al. Are patients fed appropriately according to their caloric requirements? J Parenter Enteral Nutr. 1998; 22(6): 375-81. doi: 10.1016/j.jada.2006.12.014.

13. Hoher JA, Zimermann Teixeira PJ, Hertz F, Moreira JS. A comparison between ventilation modes: how does activity level affect energy expenditure estimates? J Parenter Enteral Nutr. 2008; 32(2): 176-83. doi: 10.1177/0148607108314761.
14. Chumlea WC, Roche AF, Steinbaugh ML. Estimating stature from knee height for persons 60 to 90 years of age. J Am Geriatr Soc. 1985; 33(2):116-20.

15. Harris JA, Benedict FG. A biometric study of basal metabolism in man. Publication $n^{\circ} 279$. Washington: Carnegie Institution of Washington; 1919.

16. Van Way III CW, Ireton-Jones CS. Nutrition secrets. Philadelphia: Hanley \& Belfus; 1999.

17. Assis MCS, Silva SMR, Leães DM, Novello CL, Silveira CRM, Mello ED, et al. Nutrição enteral: diferenças entre volume, calorias e proteínas prescritos e administrado em adultos. Rev Bras Ter Intens. 2010; 22:346-50. doi: 10.1590/S0103-50 $7 \times 2010000400006$.

18. Frankenfield DC, Muth ER, Rowe WA. The HarrisBenedict studies of human basal metabolism: history and limitations. J Am Diet Assoc. 1998; 98(4):439-45. doi: 10.1016/S0002-8223(98)00 $100-\mathrm{X}$.

19. Japur CC, Penaforte FR, Chiarello PG, Monteiro JP, Vieira MN, Basile-Filho A. Harris-Benedict equation for critically ill patients: are there differences with indirect calorimetry? J Crit Care. 2009; 24(4):628 e1-5. doi: 10.1016/j.jcrc.2008.12.007.

20. Kyle UG, Genton L, Heidegger CP, Maisonneuve $\mathrm{N}$, Karsegard VL, Huber $\mathrm{O}$, et al. Hospitalized mechanically ventilated patients are at higher risk of enteral underfeeding than non-ventilated patients. Clin Nutr. 2006; 25(5):727-35. doi: 10.1 016/j.clnu.2006.03.011.

21. Whelan K, Hill L, Preedy VR, Judd PA, Taylor MA. Formula delivery in patients receiving enteral tube feeding on general hospital wards: the impact of nasogastric extubation and diarrhea. Nutrition. 2006; 22(10):1025-31. doi: 10.1016/j.nut.2006.0 7.004.

22. O'Leary-Kelley CM, Puntillo KA, Barr J, Stotts N, Douglas MK. Nutritional adequacy in patients receiving mechanical ventilation who are fed enterally. Am J Crit Care. 2005; 14(3):222-31.

23. Giner M, Laviano A, Meguid MM, Gleason JR. In 1995 a correlation between malnutrition and poor outcome in critically ill patients still exists. Nutrition. 1996; 12(1):23-9. doi: 10.1016/0899-9007(95)00 015-1.

24. Marshall AP, West SH. Enteral feeding in the critically ill: are nursing practices contributing to hypocaloric feeding? Intensive Crit Care Nurs. 2006; 22(2):95-105. doi: 10.1016/j.iccn.2005.0 9.004 .

25. Faisy C, Lerolle N, Dachraoui F, Savard JF, Abboud I, Tadie JM, et al. Impact of energy deficit calculated by a predictive method on outcome in medical patients requiring prolonged acute mechanical 
ventilation. Br J Nutr. 2009; 101(7):1079-87. doi: 10.1017/S0007114508055669.

26. Pichard C, Kyle UG, Morabia A, Perrier A, Vermeulen B, Unger P. Nutritional assessment: lean body mass depletion at hospital admission is associated with an increased length of stay. Am J Clin Nutr. 2004; 79(4):613-8.

27. Reid C. Frequency of under- and overfeeding in mechanically ventilated ICU patients: causes and possible consequences. J Hum Nutr Diet. 2006; 19(1):13-22. doi: 10.1111/j.1365-277X.2006.00 661.x.

28. Strack van Schijndel RJ, Weijs PJ, Koopmans RH, Sauerwein HP, Beishuizen A, Girbes AR. Optimal nutrition during the period of mechanical ventilation decreases mortality in critically ill, long-term acute female patients: a prospective observational cohort study. Crit Care. 2009; 13(4): R132. doi: 10.1186/cc7993.

29. Deane A, Chapman MJ, Fraser RJ, Bryant LK, Burgstad C, Nguyen NQ. Mechanisms underlying feed intolerance in the critically ill: implications for treatment. World J Gastroenterol. 2007; 13(29): 3909-17. doi: 10.1186/cc7993.

30. Elpern EH, Stutz L, Peterson S, Gurka DP, Skipper A. Outcomes associated with enteral tube feedings in a medical intensive care unit. Am J Crit Care. 2004; 13(3):221-7.

31. Nguyen NQ, Chapman MJ, Fraser RJ, Bryant LK, Burgstad C, Ching K, et al. The effects of sedation on gastric emptying and intra-gastric meal distribution in critical illness. Intensive Care Med. 2008; 34(3):454-60. doi: 10.1007/s00134-007-09 42-2.

32. Nguyen NQ, Ng MP, Chapman M, Fraser RJ, Holloway $\mathrm{RH}$. The impact of admission diagnosis on gastric emptying in critically ill patients. Crit Care. 2007; 11(1):R16. doi: 10.1186/cc5685.

33. Binnekade JM, Tepaske R, Bruynzeel P, MathusVliegen EM, de Hann RJ. Daily enteral feeding practice on the ICU: attainment of goals and interfering factors. Crit Care. 2005; 9(3):R218-25. doi: $10.1186 /$ cc3504.

34. Mutlu GM, Mutlu EA, Factor P. GI complications in patients receiving mechanical ventilation. Chest. 2001; 119(4):1222-41. doi: 10.1186/cc3504.

35. Dark DS, Pingleton SK, Kerby GR. Hypercapnia during weaning. A complication of nutritional support. Chest. 1985; 88(1):141-3. doi: 10.1378/ chest.88.1.141.

36. Japur CC, Monteiro JP, Marchini JS, Garcia RW, Basile-Filho A. Can an adequate energy intake be able to reverse the negative nitrogen balance in mechanically ventilated critically ill patients? J Crit Care. 2010; 25(3):445-50. doi: 10.1016/j.jcrc.200 9.05.009.

37. Kreymann KG, Berger MM, Deutz NE, Hiesmayr $M$, Jolliet P, Kazandjiev G, et al. ESPEN Guidelines on Enteral Nutrition: intensive care. Clin Nutr. 2006; 25(2):210-23. doi: 10.1016/j.clnu.2006.01.021.

38. Porter C, Cohen NH. Indirect calorimetry in critically ill patients: role of the clinical dietitian in interpreting results. J Am Diet Assoc. 1996; 96(1): 49-57. doi: 10.1016/S0002-8223(96)00014-4.

39. Daly JM, Heymsfield SB, Head CA, Harvey LP, Nixon DW, Katzeff $\mathrm{H}$, et al. Human energy requirements: overestimation by widely used prediction equation. Am J Clin Nutr. 1985; 42(6):1170-4.

Recebido em: 8/11/2011

Versão final em: 24/8/2012

Aprovado em: 5/9/2012 
